\title{
Evaluación de la exposición solar y las medidas de fotoprotección en deportistas del Valle de Aburrá en el 2018
}

\author{
María Soledad Aluma-Tenorio ${ }^{1}$, Jorge Alberto Osorio-Ciro², Ana María Muñoz-Monsalve, Natalia Jaimes", \\ Alejandra Ávila-Álvarez $z^{5}$ María Carolina Tamayo-Betancur ${ }^{6}$
}

\section{RESUMEN}

Antecedentes: el cáncer de piel es el más común en humanos y su incidencia continúa en aumento. La radiación ultravioleta (RUV) es el principal factor ambiental asociado con este cáncer. Los deportistas presentan un mayor riesgo para desarrollar cáncer de piel; sin embargo en nuestro país, hasta el momento, no se ha estudiado esta población.

Objetivo general: describir las conductas de riesgo del cáncer de piel en deportistas del Valle de Aburrá, a través de una encuesta realizada en los centros más importantes de la regoión.

Materiales y métodos: estudio de corte transversal descriptivo; se evaluaron variables sociodemográficas, clínicas y relacionadas con hábitos de fotoprotección. Según el tipo de variable, se usó media y desviación estándar o conteos absolutos y relativos.

Resultados: se incluyeron 122 deportistas, la edad promedio del grupo estudiado fue de 20 años, el $56 \%$ de los incluidos fueron hombres. El $74 \%$ de los deportes se realizaban al aire libre, el fútbol fue el deporte más frecuente. El $75 \%$ de los participantes entrenaba cuatro o más veces por semana y el 55 \% lo hacía en horas con muy alta RUV. EI 27 \% de los deportistas reportó de 1 a 3 insolaciones en la infancia. La fotoprotección química se utilizaba con más frecuencia que la física.

Conclusiones: este es el primer estudio realizado en Colombia que evidencia la necesidad de mejorar la educación en prevención primaria y secundaria sobre el cáncer de piel en esta población.

Cirujana de Mohs, Clínica Aurora, Medellín, Colombia.

Especialista en Medicina del Deporte, Universidad de Antioquia, Medellín, Colombia.

Dermatóloga, Clínica Aurora, Medellín, Colombia.

4 Dermatóloga, Dr Phillip Frost Department of Dermatology and Cutaneous Surgery, y Sylvester Comprehensive Cancer Center. University of Miami Miller School of Medicine. Miami, Florida, Estados Unidos.

5 Dermatóloga, Clínica Aurora, Medellín, Colombia.

6 Enfermera, Clínica Aurora, Medellín, Colombia.

Correspondencia: Alejandra Ávila Álvarez; alejavila1402@gmail.com

Recibido: noviembre 13 de 2018

Aceptado: enero 30 de 2019

Cómo citar: Aluma-Tenorio MS, Ciro-Osorio JA, Muñoz-Monsalve AM, Jaimes N, Ávila-Álvarez A, Tamayo-Betancur MC. Evaluación de la exposición solar y las medidas de fotoprotección en deportistas del Valle de Aburrá en el 2018. latreia. 2019 Jul-Sep;32(3):184-190. D0I. 10.17533/udea.iatreia.18. 


\section{PALABRAS CLAVE}

Atletas; Deportes; Neoplasias Cutáneas; Rayos UItravioleta

\section{SUMMARY}

Evaluation of solar exposure and photoprotection measures in athletes of Valle de Aburrá in 2018

Background: Skin cancer is the most common cancer in humans and its incidence increases. Ultraviolet radiation (UVR) is the main environmental factor associated with this cancer. Athletes have a greater risk to develop skin cancer. Up to date in Colombia, this population has not been studied.

Objective: To describe the behaviors and photoprotection measures in athletes of Valle de Aburrá.

Materials and methods: Cross sectional study. Sociodemographic, clinical variables and related to photoprotective habits were evaluated. Depending on the type of variable, mean and standard deviation or absolute and relative counts were used.

Results: One hundred twenty two athletes were included, $56 \%$ were men, the whole group had an average age of 20 years. $74 \%$ of sports were played outdoors; soccer was the most frequent sport. $75 \%$ of participants trained four or more times per week and 55\% did so in hours with very high RUV. 27\% of athletes reported between 1 to 3 insolations in childhood. Chemical photoprotection was used more frequently than physical.

Conclusions: This is the first study in Colombia that shows the need to improve skin cancer primary and secondary prevention strategies in this population.

\section{KEYWORDS}

Athletes; Skin Neoplasms; Sports; UItraviolet Rays

\section{INTRODUCCIÓN}

El cáncer de piel es el más común en humanos y su incidencia continúa en aumento. La radiación ultravioleta (RUV) es el principal factor ambiental asociado con este tipo de neoplasia, por lo tanto, los deportistas que entrenan al aire libre presentan un mayor riesgo de presentarla (1). A pesar de ello, no existen estudios en Colombia o Latinoamérica que evalúen la incidencia del cáncer de piel, ni los hábitos relacionados con la prevención primaria y secundaria en los deportistas.

El objetivo de este estudio es describir las características sociodemográficas, clínicas y los factores asociados a la exposición solar en distintos tipos de deportes practicados en Colombia.

\section{METODOLOGÍA}

Se realizó un estudio piloto de corte transversal descriptivo, con el fin de obtener datos iniciales para el desarrollo posterior de un estudio prospectivo analítico que incluya deportistas de diferentes ciudades de Colombia. En este estudio, se incluyeron deportistas que practican deportes organizados al aire libre y en escenarios cerrados. La investigación se realizó en diferentes instituciones deportivas y centros de entrenamiento en el Valle de Aburrá, Antioquia (Colombia).

\section{Recolección de la información y variables}

La recolección de la información se realizó a través de una encuesta desarrollada por los investigadores y entregada a cada deportista durante los entrenamientos, por médicos especialistas en medicina deportiva o médicos en formación de esta especialidad; explicando, antes de ser diligenciada por cada participante, los alcances y objetivos de este estudio.

El detalle de las variables evaluadas en la encuesta se encuentra resumido en la tabla 1.

\section{Muestreo y tamaño de muestra}

Se realizó un muestreo por conveniencia en las instituciones y centros de entrenamiento en la ciudad de Medellín: polideportivo de la Universidad EAFIT, Politécnico Colombiano Jaime Isaza Cadavid y polideportivo de la Universidad de Antioquia. El tamaño de la muestra correspondió con los deportistas que voluntariamente participaron en el estudio durante un periodo de tiempo preestablecido de un mes. No se calculó poder estadístico o error alfa por ser un estudio tipo prueba piloto. 


\section{Tabla 1. Variables evaluadas en la encuesta}

\begin{tabular}{|c|c|}
\hline Categoría & Variables \\
\hline Sociodemográficas & Sexo, edad, lugar de nacimiento y lugar de residencia \\
\hline Relacionadas con la actividad física & $\begin{array}{c}\text { Deporte practicado, inmersión acuática, tiempo de práctica del deporte, fre- } \\
\text { cuencia semanal de los entrenamientos, horario de entrenamiento, duración del } \\
\text { entrenamiento en horas, características de las áreas de entrenamiento (cubiertas o } \\
\text { expuestas) }\end{array}$ \\
\hline Clínicas & $\begin{array}{c}\text { Fototipo (se preguntó por color de pelo y piel y por la respuesta a la exposición so- } \\
\text { lar, ya sea con quemadura o bronceado), cantidad de lunares en hombros y brazos } \\
\text { (cuantificada por el mismo deportista), antecedente personal y familiar de cáncer } \\
\text { de piel e historia personal de algún síndrome genético }\end{array}$ \\
\hline $\begin{array}{c}\text { Relacionadas con la exposición } \\
\text { y protección solar }\end{array}$ & $\begin{array}{c}\text { Quemaduras solares con o sin ampollas, uso de cámaras bronceadoras, protección } \\
\text { solar química en la vida diaria y en los entrenamientos, protección solar física } \\
\text { durante los entrenamientos y autoexamen de piel }\end{array}$ \\
\hline
\end{tabular}

\section{Evaluación de ética}

A los deportistas se les solicitó el consentimiento informado verbal antes de entregarles la encuesta para su diligenciamiento.

De acuerdo con la Resolución n. ${ }^{\circ} 008430$ de 1993, que contempla las normas científicas, técnicas y administrativas para la investigación en salud, este proyecto se clasificó como investigación sin riesgo, debido a que no realiza una intervención o modificación intencionada de las variables biológicas, fisiológicas, sicológicas o sociales de los individuos que participaron en ella. No contempló, dentro de sus procedimientos, métodos, análisis de los resultados y presentación de los mismos, violación alguna de los cuatro principios de la ética biomédica contemplados en el Informe de Belmont.

\section{Análisis estadístico}

Se realizó un análisis univariado con frecuencias relativas y absolutas de las variables cualitativas. Las variables cuantitativas fueron presentadas como media y desviación estándar (DE), según la distribución normal de la variable evaluada con la prueba Kolmogorov-Smirnov. Todo esto fue realizado en Microsoft Excel, versión 2013.

\section{RESULTADOS}

Se incluyeron en el estudio 122 participantes de diferentes deportes organizados: fútbol, atletismo, halterofilia, ciclismo, voleibol, karate y tenis. La mayoría de los participantes tenían como ciudad de nacimiento y residencia a Medellín y otros municipios del Valle de Aburrá como Itagüí, Envigado, Bello, entre otros.

Se presentó un leve predominio del sexo masculino $(56 \%, \mathrm{n}=68)$, con una edad promedio de 20 años $(\mathrm{DE}=6)$. El $74 \%$ de los deportes evaluados se realizaron al aire libre, siendo el fútbol el deporte practicado con mayor frecuencia (57\%). Con respecto al tiempo que llevaba la práctica de los entrenamientos, se encontró una mediana de siete años (RIQ: 3-11,5) con un valor mínimo de dos meses y un valor máximo de 30 años (Tabla 2). El $75 \%$ de los participantes realizaban sus entrenamientos 4 veces o más a la semana, $\varnothing$ un poco más de la mitad (55\%) asistían a estos entrenamientos en horarios de máxima RUV, entre 10:00 a.m. y 3:00 p.m., con una duración promedio de 1,8 horas $(D E=0,9)$. El número de horas por semana de exposición de alto riesogo también fue calculado (Tabla 3). Solo el $7 \%$ de los participantes practicaron deportes con inmersión acuática.

Se encontró que la mayoría de los deportistas eran fototipo III (34\%), con un promedio de 0 a 20 nevus en las extremidades superiores. Un deportista (1\%) mencionó tener antecedente de cáncer de piel no melanoma y cuatro deportistas (3\%) reportaron antecedente familiar de cáncer de piel, con desconocimiento del subtipo de cáncer. El antecedente de quemadura solar fue reportado por el $58 \%$ de las mujeres; en general, el $27 \%$ de los deportistas reportaron de 1 a 3 quemaduras solares durante la infancia. 
Tabla 2. Variables sociodemográficas y clínicas de los deportistas con las características de los deportes

\begin{tabular}{|c|c|}
\hline Variables & $\begin{array}{c}\text { Número (\%) } \\
n=122\end{array}$ \\
\hline Sexo masculino & $68(56)$ \\
\hline Edad, promedio (desviación estándar) & $20(6)$ \\
\hline \multicolumn{2}{|l|}{ Color de piel } \\
\hline Blanco & $18(14,7)$ \\
\hline Mestizo & $82(67,3)$ \\
\hline Negro & $21(17,2)$ \\
\hline Sin dato & $1(0,81)$ \\
\hline \multicolumn{2}{|l|}{ Fototipo } \\
\hline I & 0 \\
\hline II & $10(8,2)$ \\
\hline III & $42(34,4)$ \\
\hline IV & $38(31,1)$ \\
\hline V & $19(15,6)$ \\
\hline VI & $13(10,6)$ \\
\hline \multicolumn{2}{|l|}{ Tipo de Deporte } \\
\hline Fútbol & $73(59,8)$ \\
\hline Karate & $10(8,2)$ \\
\hline Halterofilia & $8(6,5)$ \\
\hline Voleibol & $7(5,7)$ \\
\hline Tenis & $5(4,1)$ \\
\hline Triatlón & $5(4,1)$ \\
\hline Ciclismo/ciclocrós & $4(3,3)$ \\
\hline Atletismo & $4(3,3)$ \\
\hline Baloncesto & $2(1,6)$ \\
\hline Maratón/trote & $2(1,6)$ \\
\hline Sin dato & $2(1,6)$ \\
\hline $\begin{array}{l}\text { Tiempo de práctica del deporte (meses), } \\
\text { Mediana (RIQ) }\end{array}$ & $7(3-11,5)$ \\
\hline \multicolumn{2}{|c|}{ Frecuencia semanal de los entrenamientos } \\
\hline Menos de 4 veces por semana & $27(22,1)$ \\
\hline 4 o más veces por semana & $91(74,6)$ \\
\hline Sin dato & $4(3,3)$ \\
\hline
\end{tabular}

Tabla 3. Exposición solar de riesgo por el deporte practicado

\begin{tabular}{cc}
\hline $\begin{array}{c}\text { Deporte con reporte } \\
\text { de exposición solar de riesgo }\end{array}$ & Media horas/semana \\
Ciclismo/ciclocrós & 13 \\
\hline Fútbol & 5,4 \\
\hline Halterofilia & 4,6 \\
\hline Karate & 4,8 \\
\hline Tenis & 7,2 \\
\hline Triatlón & 4,8 \\
\hline Voleibol & 4,3 \\
\hline
\end{tabular}

En cuanto a las medidas de fotoprotección, los protectores solares tópicos fueron los más frecuentes (46\%) en comparación con las medidas de fotoprotección física como gorra, sombrero, ropa con protección solar o gafas (33 \%). Sin embargo, solo la mitad de los participantes que utilizaban protector solar tópico lo hacían durante el entrenamiento; en quienes conocían la información, el factor de protección solar reportado estaba entre 40 y 100.

EI $35 \%$ de los participantes utilizaban más de una medida de protección solar física, las más frecuentes eran las gorras y ropa con protección solar. Las mujeres utilizaban con más frecuencia la protección solar química ( $55 \%, \mathrm{n}=28$ ), mientras que en los hombres prevalecía la protección solar física $(55 \%, \mathrm{n}=22)$.

El uso de protección solar química fue similar en el fútbol y los otros deportes ( $41 \%$ en fútbol y $43 \%$ en otros deportes). Como era de esperarse, por los implementos aceptados para uso en el fútbol, la protección solar física fue menor en estos deportistas (26\% en los futbolistas, $42 \%$ en los otros deportes). En general, los deportistas que realizaron sus entrenamientos a la sombra o bajo techo reportaron menor uso de protección solar física (28\% frente a $36 \%$ ) y química (25\% frente a $48 \%$ ) en comparación con los participantes que realizaban los deportes al aire libre (Tabla 4).

Solo cuatro deportistas ( 3 \%) tenían conocimiento sobre cómo realizar el autoexamen de piel, y de estos, menos del 2 \% realizaban en su autoexamen de piel el ABCDE para detección de melanoma. Estos cuatro deportistas presentaban en común el uso de protección solar física y química durante los entrenamientos, no tenían antecedente de quemaduras solares en 
la infancia y pertenecían a diferentes grupos de deportes como fútbol, karate y halterofilia. Ningún participante mencionó realizar el autoexamen de piel de forma mensual (Tabla 4).

Tabla 4. Características relacionadas con la exposición y las medidas de protección solar

\begin{tabular}{|c|c|}
\hline $\begin{array}{l}\text { Variables relacionadas con la exposición } \\
\text { solar y fotoprotección }\end{array}$ & $\begin{array}{l}\text { Número (\%) } \\
n=122\end{array}$ \\
\hline \multicolumn{2}{|l|}{ Horario de entrenamiento } \\
\hline Antes de las 10 a.m. & $13(10,6)$ \\
\hline De 10 a.m. a 3 p.m. & $67(55,0)$ \\
\hline Otros horarios & $42(34,4)$ \\
\hline \multicolumn{2}{|c|}{ Tipo de protección solar usada en el entrenamiento } \\
\hline Química & $56(46,0)$ \\
\hline Física & $40(32,8)$ \\
\hline \multicolumn{2}{|l|}{ Factor de protección solar } \\
\hline No uso de protector solar & $66(54,1)$ \\
\hline $\begin{array}{l}\text { Uso, pero desconocimiento de las caracte- } \\
\text { rísticas }\end{array}$ & $36(29,5)$ \\
\hline FPS 45 & $1(0,8)$ \\
\hline FPS $50+$ & $19(15,6)$ \\
\hline \multicolumn{2}{|l|}{ Medidas de protección física } \\
\hline Gorra & $20(16,4)$ \\
\hline Ropa con protección solar & $10(8,2)$ \\
\hline Gafas & $3(2,4)$ \\
\hline Sombrero & $1(0,8)$ \\
\hline Sin dato & $6(4,9)$ \\
\hline \multicolumn{2}{|l|}{ Conocimiento del autoexamen de piel } \\
\hline No & $117(96,0)$ \\
\hline Sí & $4(3,2)$ \\
\hline Sin respuesta & $1(0,8)$ \\
\hline \multicolumn{2}{|l|}{ Práctica del autoexamen de piel } \\
\hline No & $92(75,4)$ \\
\hline Sin respuesta & $30(24,6)$ \\
\hline
\end{tabular}

El $66,4 \%(n=81)$ reportaron conocer los posibles efectos de la exposición solar en la piel durante el entrenamiento y el 34 \% reportaron la realización de actividades para fomentar el cuidado de su piel durante el entrenamiento, por parte de sus entrenadores. Estas actividades fueron más frecuentes en los futbolistas, comparado con los otros deportes.

El $39 \%$ de los participantes del estudio presentaron fototipo altos: IV, $\nabla$ y VI. De estos, el $65 \%$ practicaban fútbol. En este subgrupo no se presentaron diferencias que pudieran compararse con la totalidad de los participantes con respecto a los horarios y duración de los entrenamientos o el uso de protección solar química. Solo dos de estos deportistas presentaron antecedente de quemaduras solares. Con respecto a la protección solar física, se utilizó una menor proporción en este grupo ( $21 \%$ en fototipos altos, $33 \%$ en todos los participantes).

\section{DISCUSIÓN}

El cáncer de piel es el más frecuente en el mundo y su incidencia ha incrementado en las últimas tres décadas. Para Colombia, según las cifras de los casos atendidos en el Instituto Nacional de Cancerología, hay un aumento continuo del número de casos de cáncer de piel desde 1996, siendo hasta el 2010, el 23,3 \% de todos Ios tipos de cáncer, prácticamente el doble de los de cáncer de mama y de cuello uterino, y de cuatro a cinco veces más que los tumores de la próstata, el sistema hematolinfoide y el sistema gastrointestinal (1).

La RUV es el principal factor ambiental asociado con las neoplasias malignas cutáneas. Por lo tanto, los deportistas que entrenan al aire libre presentan un mayor riesgo para desarrollar esta condición. Este es el primer estudio realizado en Colombia, en conocimiento de los autores, sobre las prácticas relacionadas con la fotoprotección en deportistas y la caracterización del riesgo a desarrollar cáncer de piel. Este estudio refleja algunas de las prácticas deportivas más frecuentes en Medellín, a diferencia de estudios realizados en otras regiones que incluyen deportes como hockey, esquí y montañismo (2), los cuales son muy poco frecuentes en nuestro país.

Las características sociodemográficas observadas en nuestro estudio fueron similares a lo reportado en 
otras investigaciones $(2,3)$. Se encontró una mediana de entrenamiento de siete años (RIQ: 3-11,5), con un valor mínimo de dos meses y un valor máximo de 30 años, iniciando sus primeras practicas a edades tempranas. Esto es importante, en especial en aquellos deportes que se realizan al aire libre, pues se conoce que en las primeras dos décadas de la vida se acumula aproximadamente un 22,7\% de la RUV que se recibirá durante toda la vida. El conocimiento de esto permitiría la implementación de medidas de fotoprotección desde edades tempranas con el objetivo de reducir la exposición a la RUV y, por lo tanto, el riesgo para desarrollar cáncer de piel en el futuro (4).

Se evidenció que más de la mitad de los participantes realizan sus entrenamientos al aire libre, con una frecuencia de cuatro o más veces a la semana y en el horario que comprende de 10 a.m. a 3:00 p.m. Este período de tiempo es considerado el de mayor RUV, ya que los rayos solares llegan de forma perpendicular y no paralela a la superficie terrestre, lo cual facilita su profundización en la piel (5-7). Adicionalmente, debido a su localización geográfica entre los trópicos y altitud, la ciudad de Medellín presenta altos niveles de RUV durante todo el año (8). Lo anterior explica por qué la práctica del deporte al aire libre en estos horarios se considera un factor de riesgo para desarrollar cáncer de piel.

EI $73 \%$ de los deportistas presentaban fototipos del III aI $\mathrm{VI}$, lo que refleja las características del mestizaje de la población colombiana y diferencia este estudio de otros publicados con poblaciones de piel más clara (2, 3, 9-11). Esto a su vez podría explicar el hecho de que menos de una tercera parte del grupo tuviera el antecedente de quemaduras en la niñez, ya que por el tipo de piel, es más frecuente la presencia de bronceado y no de quemaduras o ampollas en esta población, lo que podría, en teoría, reducir la asociación ya conocida de quemadura solar en la niñez y melanoma (12-15).

En este estudio no se reporta el antecedente de uso de cámaras de bronceo, a pesar de que la población incluida, se encuentra en el rango de edad que más se expone a dichas cámaras en otros países (16).

A pesar de que dos terceras partes de los deportistas reportaron conocer los posibles efectos de la RUV sobre la piel, se observó poca adherencia a las medidas de fotoprotección, tanto químicas como físicas y, en general, un desconocimiento sobre el autoexamen de piel. Lo anterior resalta la importancia de la educación en la prevención primaria y secundaria en los grupos de alto riesgo como los deportistas al aire libre.

Finalmente, se destaca el trabajo interdisciplinario con deportólogos y dermatólogos, lo que permitió identificar el perfil de riesogo para desarrollar cáncer de piel en los deportistas de nuestro país.

Las limitaciones del estudio incluyeron la forma de recolección de la información, en la que los participantes pueden sentirse evaluados y modificar algunas de sus respuestas o emitir algunas equivocadas acerca de las variables como el fototipo, las quemaduras solares y el conteo de nevus.

Aunque el diseño de tipo corte transversal podría considerarse como una limitante, estos resultados iniciales permitirán realizar un estudio posterior de tipo prospectivo con el objetivo de hacer inferencias sobre el riesgo específico del cáncer de piel en los deportistas que realizan sus entrenamientos al aire libre.

\section{CONCLUSIONES}

Los deportistas del Valle de Aburrá tienen una alta exposición a la RUV y en un número importante de casos no hay un uso adecuado de las medidas de fotoprotección, así como un desconocimiento general del autoexamen de piel.

Este estudio arroja datos iniciales que demuestran la necesidad de mejorar la educación en la prevención primaria y secundaria en este grupo poblacional.

\section{CONFLICTOS DE INTERESES}

Ninguno por declarar.

\section{REFERENCIAS BIBLIOGRÁFICAS}

1. Pozzobon FC, Acosta AE, Castillo JS. Cáncer de piel en Colombia: cifras del Instituto Nacional de Cancerología. Revista asociación colombiana de dermatología. 2018;26(1):12-17.

2. Laffargue JA, Merediz J, Buján MM, Pierini AM. Sun protection questionnaire in Buenos Aires adolescent 
athletes. Arch Argent Pediatr. 2011 Feb;109(1):30-5. DOI 10.1590/S0325-00752011000100008.

3. Moehrle M. Outdoor sports and skin cancer. Clin Dermatol. 2008 Jan-Feb;26(1):12-5. DOI 10.1016/j.clindermatol.2007.10.001.

4. Falk M, Anderson CD. Influence of age, gender, educational level and self-estimation of skin type on sun exposure habits and readiness to increase sun protection. Cancer Epidemiol. 2013 Apr;37(2):127-32. DOI 10.1016/j.canep.2012.12.006.

5. Lawler S, Spathonis K, Eakin E, Gallois C, Leslie E, Owen N. Sun exposure and sun protection behaviours among young adult sport competitors. Aust N Z J Public Health. 2007 Jun;31(3):230-4. DOI https:// doi.org/10.1111/j.1467-842X.2007.00053.x.

6. Jinna S, Adams BB. Ultraviolet radiation and the athlete: risk, sun safety, and barriers to implementation of protective strategies. Sports Med. 2013 Jul;43(7):531-7. DOI 10.1007/s40279-013-0021-5.

7. Holick MF. Biological Effects of Sunlight, Ultraviolet Radiation, Visible Light, Infrared Radiation and Vitamin D for Health. Anticancer Res. 2016 Mar;36(3):1345-56.

8. Índice UV [internet]. Bogotá: MINAMBIENTE; c2011. [Consultado 2018 sep 11]. Disponible en: http://bart. ideam.gov.co/wrfideam/indiceuv/

9. Christoph S, Cazzaniga S, Hunger RE, Naldi L, Borradori L, Oberholzer PA. Ultraviolet radiation protection and skin cancer awareness in recreational athletes: a survey among participants in a running event. Swiss Med Wkly. 2016 Mar 21;146:W14297. DOI 10.4414/smw.2016.14297.
10. Falk M, Anderson CD. Influence of age, gender, educational level and self-estimation of skin type on sun exposure habits and readiness to increase sun protection. Cancer Epidemiol. 2013 Apr;37(2):127-32. DOI 10.1016/j.canep.2012.12.006.

11. Azoury SC, Lange JR. Epidemiology, risk factors, prevention, and early detection of melanoma. Surg Clin North Am. 2014 Oct;94(5):945-62. DOI 10.1016/j. suc.2014.07.013.

12. Taylor NJ, Thomas NE, Anton-Culver H, Armstrong BK, Begg CB, Busam KJ. Nevus count associations with pigmentary phenotype, histopathological melanoma characteristics and survival from melanoma. Int J Cancer. 2016 Sep 15;139(6):1217-22. DOI 10.1002/ ijc.30157.

13. Sturm RA. Skin colour and skin cancer-MC1R, the genetic link. Melanoma Res. 2002 Oct;12(5):405-16.

14. Aluma-Tenorio MS, Ávila-Álvarez A, Jaimes N, CabaIlero-Uribe N, González A, Terzian LR, et al. Basal cell carcinoma treated with Mohs micrographic surgery in young Ibero-American patients. Int J Dermatol. 2018 Aug 31. DOI 10.1111/ijd.14195.

15. Gupta AK, Bharadwaj M, Mehrotra R. Skin Cancer Concerns in People of Color: Risk Factors and Prevention. Asian Pac J Cancer Prev. 2016 Dec 1;17(12):525764. DOI 10.22034/APJCP.2016.17.12.5257.

16. Wehner MR, Chren MM, Nameth D, Choudhry A, Gaskins M, Nead KT, et al. International prevalence of indoor tanning: a systematic review and metaanalysis. JAMA Dermatol. 2014 Apr;150(4):390-400. DOI 10.1001/jamadermatol.2013.6896. 